\title{
Outcome of open reduction and Kirschner wire fixation in pediatric radial neck fracture
}

\author{
Alireza Rouhani ${ }^{1}$, Mohammadreza Chavoshi ${ }^{2}$, Alireza Sadeghpour ${ }^{3}$, Hossein Aslani ${ }^{4}$, \\ Mohsen Mardani-Kivi ${ }^{5}$ \\ ${ }^{1}$ Department of Orthopedics, Shohada Hospital, Tabriz University of Medical Sciences, Tabriz, Iran \\ ${ }^{2}$ Department of Radiology, Tehran University of Medical Sciences, Tehran, Iran \\ ${ }^{3}$ Department of Orthopedic Surgery, School of Medicine and Shohada Educational Hospital, Tabriz University of Medical Sciences, Tabriz, Iran \\ ${ }^{4}$ Department of Anesthesiology, Tabriz University of Medical Sciences, Tabriz, Iran \\ ${ }^{5}$ Orthopedic Research Center, Department of Orthopedics, Poursina Hospital, School of Medicine, Guilan University of Medical Sciences, Rasht, Iran
}

\begin{abstract}
Background: Radial neck fracture in children is rare. This study attempted to evaluate the outcome of surgically treated patients and any associated complications.

Methods: This study evaluated 23 children under 15 years of age with radial neck fracture who were treated with open reduction between 2006 and 2016 to determine their range of motion, postoperative complications, and radiographic outcomes. The results were assessed clinically using the Mayo clinic elbow performance score.

Results: The mean follow-up duration for patients was 34.6 months. The average postoperative angulation was $3.6^{\circ}$. Hypoesthesia was reported in only $9 \%$ of patients, and none of the patients complained of postoperative pain. The postoperative X-ray results were excellent in $60 \%$ and good in $40 \%$. No radiographic complications were identified. The elbow score was excellent in $87 \%$ and good in $13 \%$ (mean score, 96.74). There was a statistical relationship between range of motion limitations and age, degree of fracture, initial displacement, and surgical pin removal time.

Conclusions: Although most patients accept the closed reduction method as a primary treatment, the present study suggests that an open-reduction approach has been associated with optimal therapeutic outcomes for patients in whom closed reduction was not satisfactory or indicated.
\end{abstract}

Keywords: Radius; Open fracture reduction; Radius fractures; Pediatrics; Treatment outcome

\section{INTRODUCTION}

Radial neck fracture in children is a relatively rare injury and comprises up to $10 \%$ of elbow fractures and $5 \%$ of all pediatric fractures [1,2]. In most cases, the mechanism of fracture is falling on the arm while the elbow is in extension [3,4]. The presence of radial head blood supply impairment after fracture can lead to avascular necrosis of the radial head and some other complica-

Received: June 22, $2021 \quad$ Revised: September 29, $2021 \quad$ Accepted: October 4, 2021

Correspondence to: Mohsen Mardani-Kivi

Orthopedic Research Center, Department of Orthopedics, Poursina Hospital, School of Medicine, Guilan University of Medical Sciences, Rasht

4137816357, Iran

Tel: +98-912-3544365, Fax: +98-13-33311178, E-mail: Mardani.kivi@gmail.com, ORCID: https://orcid.org/0000-0002-9437-5756

Financial support: None.

Conflict of interest: None.

Copyright@ 2021 Korean Shoulder and Elbow Society.

This is an Open Access article distributed under the terms of the Creative Commons Attribution Non-Commercial License (http://creativecommons.org/licenses/by-nc/4.0/) which permits unrestricted non-commercial use, distribution, and reproduction in any medium, provided the original work is properly cited. 
tions, such as closure of the epiphyseal plate, nonunion, synostosis, infection, and limited range of motion (ROM) [5-7]. The median age of children who sustain this type of injury is 9-10 years, and no difference in sex prevalence was seen $[1,3,5]$.

Controversies about the best treatment of these fractures (especially in angulated or displaced fractures) remain [8]. Recent studies have relied on the closed reduction technique as a cornerstone of treatment because of the high rate of side effects reported for open reduction methods $[1,3,5,6]$. However, open reduction is unavoidable in some cases. Generally, open reduction techniques are used in patients with severe angulation or displacement, multiple fractures of the elbow, and failure of closed reduction $[3,7,9]$. Although previous studies have emphasized the high rate of complications that occur with open reduction techniques, our experiments showed the opposite. This study attempted to evaluate the outcome of surgically treated patients and determine the prevalence of complications with this treatment.

\section{METHODS}

The study was approved by the research ethics committee of Tabriz University of Medical Sciences (No. IR.TBZMED.VCR.REC. 1397.143). Written informed consent was obtained from the patients' parents for their anonymized information to be published in this article.

This was a cross-sectional study conducted through evaluation of the medical records and follow-up visit summaries of patients who were admitted with a confirmed diagnosis of radial neck fracture to an orthopedic center between March 2006 and September 2016. Data for all patients with a definite diagnosis of radial neck fracture and who were younger than 15 years at the time of fracture were extracted. The diagnosis of radial neck fracture was confirmed by conventional radiography or computed tomography scanning of the elbow, and these images were interpreted by two orthopedic surgeons (a pediatric orthopedic surgeon and a shoulder and elbow orthopedic surgeon). All cases treated with nonoperative methods, closed reduction, or closed reduction and percutaneous fixation were excluded from the study, and only patients who needed open reduction and internal fixation were included in this investigation. Furthermore, patients were excluded if the diagnosis was uncertain, the patient was older than 15 years, medical records were missing, or if follow-up was not possible. Data regarding age at fracture, type of fracture according to Judet's classification, angular displacement, associated fracture, mechanism of injury, duration of immobilization, pin removal time after surgery, postoperative clinical ex- amination (including ROM, neural examination, and postsurgical complications), and postsurgical radiographic results were extracted from the patient medical records. After collecting the data, all included patients were asked to participate in an in-person examination, which was carried out with all except one participant who was not able to come to the orthopedic clinic due to the great distance. The data for this patient were obtained via a telephone interview; to assess elbow motions, this patient was asked to take directed photos of his elbow. Among 251 patients, 184 were treated nonoperatively, 45 were treated using closed methods, and 22 (11 females and 11 males) fulfilled the inclusion criteria and entered the study. One of the female patients had bilateral fractures, so we considered her as two cases.

All cases were operated on by two orthopedic surgeons (a pediatric orthopedic surgeon and a shoulder and elbow orthopedic surgeon) at an orthopedic referral hospital with one technique. The average duration of the follow-up period was $34.6 \pm 5.6$ months (range, 6-96 months).

The Mayo clinic elbow performance score was used to evaluate the function of the elbow [10]. Post-surgical radiographs were classified using the Metaizeau classification [11]. Our cutoff for a normal ROM was based on the study of the American Academy of Orthopedic Surgeons (flexion, 146; supination, 84; extension, 0 ; pronation, 71). After induction of general anesthesia, closed reduction or closed percutaneous reduction was attempted under control of an image intensifier. If there was $>30^{\circ}$ of angulation, $>3 \mathrm{~mm}$ translation, or an associated fracture, we continued with open reduction.

The Kocher approach was used in all cases. Under tourniquet control, the incision was centered over the lateral epicondyle and extended proximally over the lateral ridge and distally parallel to the ulna. Dissection was continued between the anconeus and extensor carpi ulnaris muscles; when the proximal end of the radius was visible, the radial head was manipulated, and the forearm was rotated to reduce the fracture and was pinned with two $1.5-\mathrm{mm}$ cross or parallel pins. After surgery, the elbow was immobilized for 4 weeks with a splint that was removed once a day for elbow motion. No forearm rotation was allowed until pin removal. Pins were removed 4-16 weeks after surgery depending on the postoperative radiographs.

The recorded information was imported to the IBM SPSS ver. 22.0 (IBM Corp., Armonk, NY, USA). A normal distribution of the quantitative data was assessed using the Shapiro-Wilk test, and if the normality was rejected, a Mann-Whitney U-test (nonparametric) was used. The data are expressed as mean \pm standard deviation or median (interquartile range), as appropriate. A multivariate analysis was designed to predict the risk factors related 
to the quantitative data. A Spearman test was used for continuous variables with a normal distribution. Binomial variables were analyzed with a chi-square analysis. The null hypotheses were rejected if the p-value was $<0.05$.

\section{RESULTS}

The mean age of the patients was $9.09 \pm 0.46$ years (range, $1-12$ years). The mean age of girls was $9.08 \pm 0.63$ years, while the average age of boys was $9.09 \pm 0.69$ years. Fifteen fractures $(65.2 \%)$ were located on the right side, and eight (34.8\%) were found on the left side. In 21 patients $(95.7 \%)$, the mechanism of the fracture was fall onto the outstretched arm, and only one fracture (4.3\%) was due to a car accident. The average angulation at admission was $54.4^{\circ} \pm 3.3^{\circ}$. Based on the Judet classification, two patients $(8.7 \%)$ had $<30^{\circ}$ angulation (type 2), 20 (87\%) demonstrated $30^{\circ}-60^{\circ}$ angulation (type 3 ), and one (4.3\%) had $>60^{\circ}$ angulation (type 4 ). Both patients with Judet type 2 fracture also had an associated ulnar fracture (Fig. 1). No patient was diagnosed with a type 1 fracture. Eleven patients (47.8\%) had an associated fracture (ulnar shaft, 5; radial head, 3; monteggia, 2; distal radius, 2; olecranon, 1 ).

Among these 11 patients, 8 had a type 3 radial neck fracture, 2 had a type 2 radial neck fracture, and 1 patient's fracture was type 4. The average length of immobilization after surgery was $5.5 \pm 1$ days (range, 0-10 days). The mean time until pin removal after surgery was 6.5 weeks (range, 4-16 weeks). The average length of treatment (from the first day of admission until pin removal) was 51.2 days. Postsurgical radiographs were interpreted based on the Metaizeau classification; in 14 patients (60\%), the result was excellent, while 9 patients (40\%) experienced good results. The mean angulation after surgery was $3.6^{\circ} \pm 1.1^{\circ}$. Postsurgical radiographs were examined for complications, including avascular necrosis of the radial head, non-union, radioulnar synostosis, degenerative changes in the elbow, heterotopic ossification, and premature epiphyseal closure. No complications were seen on radiographic evaluation. During follow-up examinations, only two patients (9\%) complained of hypoesthesia in the radial nerve area, and both improved after 2 weeks without medical intervention. One patient complained of muscle weakness that improved after physiotherapy. Pain with motion after surgery was not reported by any of the patients (Table 1). Upon clinical examination, 16 patients $(69.9 \%)$ had limitation in supination/pronation, and five $(21.7 \%)$ demonstrated limitation in flexion/extension. The limitations in pronation in five patients (22\%) and in supination in 10 patients $(44 \%)$ were $>20^{\circ}$.

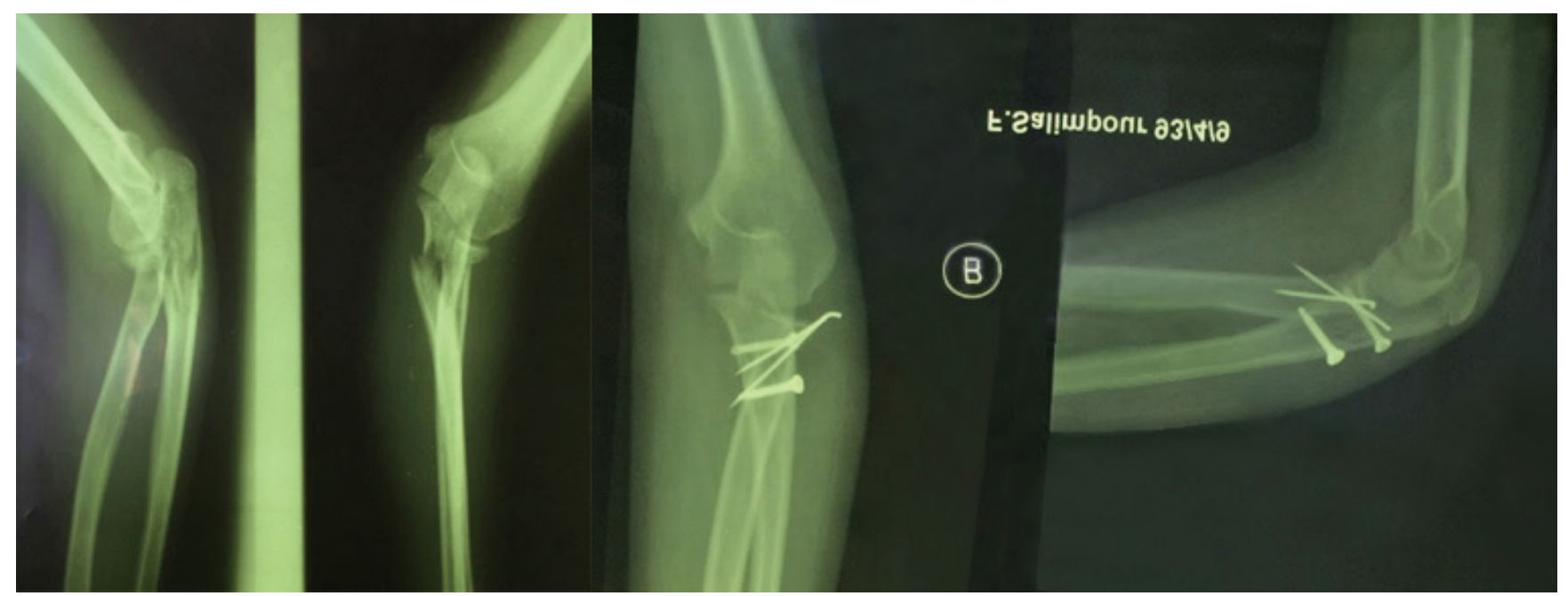

Fig. 1. After surgery.

Table 1. Postoperative forearm range of motion

\begin{tabular}{lcccc}
\hline Variable & Flexion $\left(^{\circ}\right)$ & Extension $\left(^{\circ}\right)$ & Supination $\left(^{\circ}\right)$ & Pronation $\left(^{\circ}\right)$ \\
\hline Mean & 141.30 & 2.39 & 65.43 & 61.74 \\
Median & 145 & 0.00 & 70 & 65 \\
Max & 145 & 20 & 80 & 85 \\
Min & 130 & -10 & 40 & 40 \\
\hline
\end{tabular}




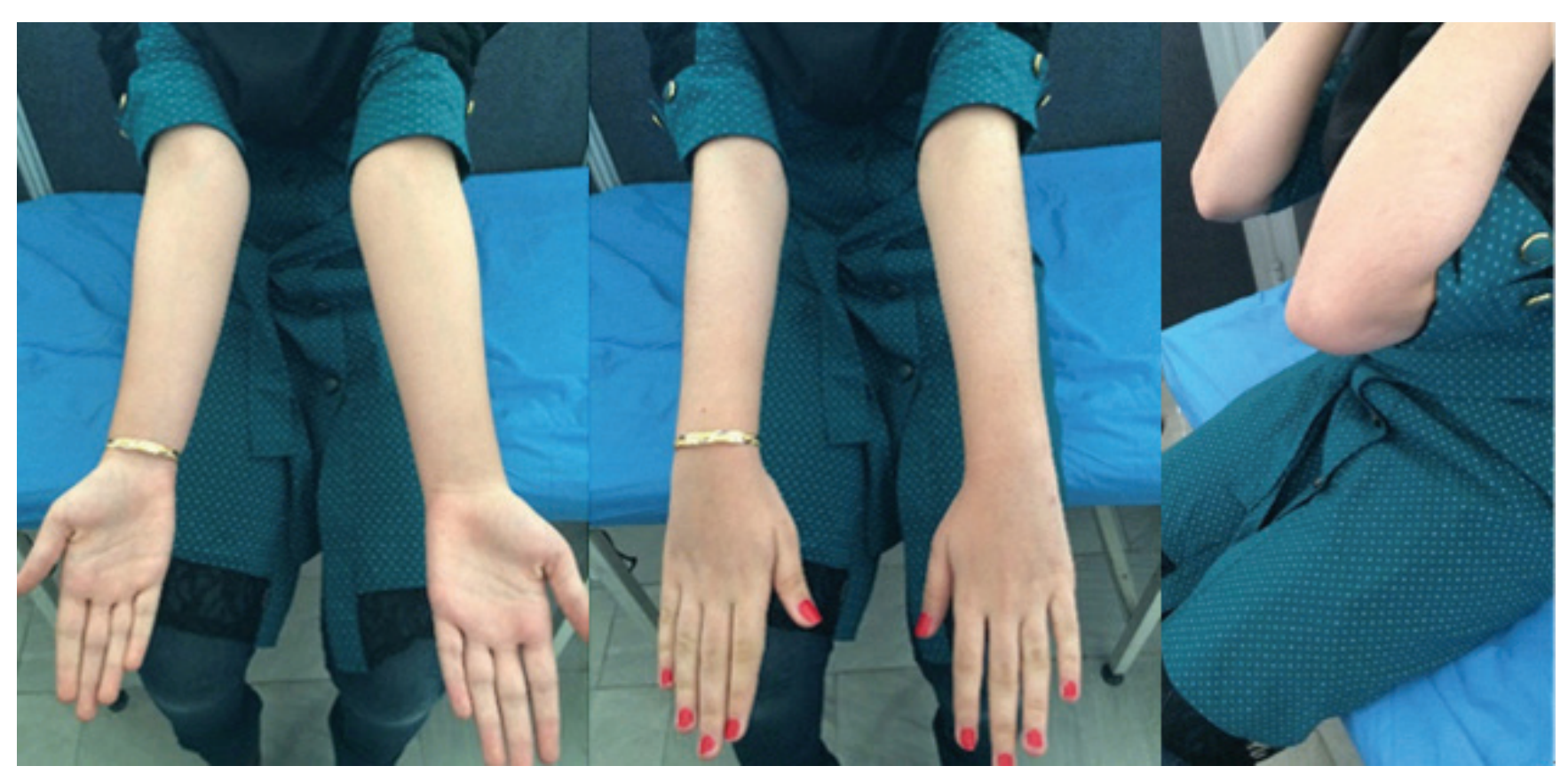

Fig. 2. Range of motion after pin removal.

Table 2. Spearman correlations between variables and elbow movements

\begin{tabular}{lcccc}
\hline \multirow{2}{*}{ Variable } & \multicolumn{3}{c}{ Spearman correlation (p-value) } \\
\cline { 2 - 5 } & Flexion & Extension & Supination & Pronation \\
\hline Post-surgery angulation & $-0.62(0.002)$ & $0.64(0.001)$ & $-0.56(0.005)$ & $-0.61(0.002)$ \\
Immobilization & $0.21(0.34)$ & $-0.16(0.94)$ & $-0.45(0.03)$ & $-0.20(0.35)$ \\
Pin removal & $-0.42(0.045)$ & $0.44(0.035)$ & $-0.57(0.005)$ & $-0.56(0.005)$ \\
Age & $-0.54(0.01)$ & $0.68(0.00)$ & $-0.57(0.004)$ & $-0.49(0.017)$ \\
First angulation & $-0.05(0.82)$ & $0.09(0.68)$ & $-0.70(0.00)$ & $-0.61(0.002)$ \\
Displacement & $0.12(0.58)$ & $0.19(0.39)$ & $-0.48(0.02)$ & $-0.36(0.095)$ \\
\hline
\end{tabular}

According to the Mayo elbow performance score, 20 patients (87\%) received excellent and three (13\%) received a good score (mean score, 96.74). According to the Spearman correlation between different variables and ROM of each elbow movement, there was a strong negative correlation between age and ROM $(\mathrm{p}<0.05)$ (Fig.2). A negative correlation was seen between pin removal and ROM. In addition, our findings indicated a negative correlation between first angular displacement and ROM $(\mathrm{p}<0.05)$. A negative correlation also existed between duration of immobilization and supination $\left(\mathrm{r}_{\mathrm{s}}=-0.45, \mathrm{p}<0.05\right)$ (Table 2).

A chi-square test of independence was performed to examine the relationship between associated fracture and limited ROM with supination/pronation and flexion/extension. The relationship between these variables was not significant; $\left[\chi^{2}(2, N=11)=0.1\right.$, $\mathrm{p}=0.75$ and $\chi^{2}(2, \mathrm{~N}=11)=0.1, \mathrm{p}=0.69$, respectively]. There was no significant association between sex and limited ROM in supination/pronation and flexion/extension $\left[\chi^{2}(2, \mathrm{~N}=23)=1.5\right.$, $\mathrm{p}=0.22$ and $\chi^{2}(2, \mathrm{~N}=23)=0.1, \mathrm{p}=0.69$, respectively $]$

\section{DISCUSSION}

Although pediatric radial neck fracture is uncommon, this injury can cause serious complications due to its effect on forearm rotation and elbow motion. The reported average age of patients is 9-10 years [6]. In our study, the average age was 9 years, and there was no age difference between boys and girls.

Due to the high rate of bone remodeling in children, the standard method of treatment for radial neck fracture with angulation $<30^{\circ}$ and displacement $<2 \mathrm{~mm}$ is closed reduction [12-17]. Some articles have suggested that closed reduction can be the only therapy for angulations up to $45^{\circ}[1,3,5,6]$. However, in a study published in 1998, Vocke and Von Laer [12] proposed that multiple tries at closed reduction cause muscular stiffness, bleeding, and additional injury to the joint. Another study from 1993 
by Metaizeau et al. [11] revealed that treatment of fracture only with closed reduction can increase the risk of secondary displacement. Closed reduction was considered for all patients in this study. If the result was not satisfactory, if the angulation was $>30^{\circ}$, or if there was $>3 \mathrm{~mm}$ translation, closed reduction was not a suitable choice, so the patient underwent open reduction.

One of the most important complications of therapy in pediatric radial neck fracture that is considered in almost all studies is limited ROM. Some studies have mentioned the high incidence of limited ROM with the open reduction technique [18]. In 1993, Metaizeau et al. [11] proposed that $40 \%-45 \%$ of type 3 and 4 fractures had a poor to moderate outcome or ROM after surgery. A newer study from 2016 compared the results of open vs. closed reduction. Of the 68 patients who underwent surgery, 14 (21\%) experienced limited ROM [8]. In contrast, Steinberg et al. [6] showed that better treatment results were seen in surgically treated patients instead of in the non-surgically treated group. In a study published in 2011 by Tan and Mahadev [19] in Singapore, the age of the patient was important in treatment outcome, and older ages caused poorer outcomes. Other studies have mentioned poorer outcomes in older children [3,20,21]. Similarly, our study found that increasing patient age increased the risk of limited ROM. This result can be attributed to the higher rate of remodeling at younger ages.

In our study, $69.6 \%$ of patients had a few degrees of ROM limitation. Except for age, this limitation was related to other matters, like initial angulation degree, post-surgical angulation, and pin removal time, while variables like sex, associated fracture, duration of immobilization after surgery, and initial displacement had no significant correlation. As shown in other studies $[20,21]$, we found that the initial angulation of the bone played an important negative role in the outcome of treatment. One interesting point was that, in our study, this correlation was seen only for supination/pronation; the range of flexion/extension was not affected. Previous studies have mentioned that the rate of the displacement can influence the outcome and ROM [6,11]. Although none of them specifically mentioned the most affected movement of the elbow, our study showed a weak correlation only for supination ( $\left.\mathrm{p}=0.2, \mathrm{r}_{\mathrm{s}}=-0.48\right)$.

Although not mentioned in previous studies, we noted a relatively strong correlation between postsurgical ROM and pin removal time. When the pin removal time was increased, all four movements of the elbow and forearm were limited, indicating that early removal allows earlier joint movements and could reduce the rate of postsurgical complications. However, pin removal time can influence the stability of the bone after reduction. Since this variable was varied in other studies, additional study should focus on this issue to determine the optimal time for pin removal. Radial head deformity is a complication that results from both closed and open reduction methods $[3,13,22]$. In previous studies, the prevalence of this complication has ranged from $12 \%$ [13] to $83 \%$ [12]; in our study, there was only one case (4\%) with this complication. Early closure of the epiphyseal plate is another complication that can arise and has a prevalence ranging from $9 \%$ [1] to 50\% [3], but there was no case in our study. Radioulnar synostosis is another important complication in surgically treated patients, with a prevalence up to $10 \%$ [3]. Our sample population did not include any patients with this complication. It seems that the prevalence of these complications is related to both the method of treatment and the surgical technique.

In children and adolescents, the functional ROM for the elbow to carry out contemporary tasks is $51^{\circ}-139^{\circ}$ flexion/extension and $18^{\circ}-55^{\circ}$ supination/pronation [23]. This finding indicates that children do not require full ROM to perform activities. This result is in accordance with our study findings that, while nearly $70 \%$ of our patients had limited ROM upon clinical examination, $87 \%$ of them obtained an excellent score on the Mayo Elbow Performance Scale, and $13 \%$ had a good score. Therefore, to achieve a good practical result of treatment, it is not necessary to fixate on the degree of ROM.

This study was conducted at a single tertiary orthopedic center with 23 patients. The small number of cases and the lack of a control group were the main limitations of this study. Since this was a retrospective study, only patients who had data available and were referred for follow-up examinations were included. In addition, any variation in immobilization period and pin removal time reduced the accuracy of analysis. Different postoperative care techniques between patients might have affected the final results.

In conclusion, despite the high prevalence of complications reported for the open reduction method in previous studies, many of these complications were either not observed in our study or the prevalence was lower. Therefore, although clinical examination might have indicated a limited ROM in many patients, this difference did not lead to significant functional problems.

\section{ORCID}

Alireza Rouhani https://orcid.org/0000-0003-0890-2001 Mohammadreza Chavoshi https://orcid.org/0000-0001-6598-7190 Alireza Sadeghpour https://orcid.org/0000-0003-0585-4470 Hossein Aslani https://orcid.org/0000-0003-2793-0980 Mohsen Mardani-Kivi https://orcid.org/0000-0002-9437-5756 


\section{REFERENCES}

1. D'souza S, Vaishya R, Klenerman L. Management of radial neck fractures in children: a retrospective analysis of one hundred patients. J Pediatr Orthop 1993;13:232-8.

2. Ursei M, Sales de Gauzy J, Knorr J, Abid A, Darodes P, Cahuzac JP. Surgical treatment of radial neck fractures in children by intramedullary pinning. Acta Orthop Belg 2006;72:131-7.

3. Newman JH. Displaced radial neck fractures in children. Injury 1977;9:114-21.

4. O'Brien PI. Injuries involving the proximal radial epiphysis. Clin Orthop Relat Res 1965;41:51-8.

5. Vahvanen V, Gripenberg L. Fracture of the radial neck in children: a long-term follow-up study of 43 cases. Acta Orthop Scand 1978;49:32-8.

6. Steinberg EL, Golomb D, Salama R, Wientroub S. Radial head and neck fractures in children. J Pediatr Orthop 1988;8:35-40.

7. Flynn JM, Skaggs DL, PM W. Rockwood and Wilkins' fractures in children. 8th ed. Netherlands: Lippincott Williams \& Wilkins; 2014. p. 474-6.

8. De Mattos CB, Ramski DE, Kushare IV, Angsanuntsukh C, Flynn JM. Radial neck fractures in children and adolescents: an examination of operative and nonoperative treatment and outcomes. J Pediatr Orthop 2016;36:6-12.

9. Rodriguez Merchan EC. Displaced fractures of the head and neck of the radius in children: open reduction and temporary transarticular internal fixation. Orthopedics 1991;14:697-700.

10. Turchin DC, Beaton DE, Richards RR. Validity of observer-based aggregate scoring systems as descriptors of elbow pain, function, and disability. J Bone Joint Surg Am 1998;80:154-62.

11. Metaizeau JP, Lascombes P, Lemelle JL, Finlayson D, Prevot J. Reduction and fixation of displaced radial neck fractures by closed intramedullary pinning. J Pediatr Orthop 1993;13:35560.
12. Vocke AK, Von Laer L. Displaced fractures of the radial neck in children: long-term results and prognosis of conservative treatment. J Pediatr Orthop B 1998;7:217-22.

13. Jones ER, Esah M. Displaced fractures of the neck of the radius in children. J Bone Joint Surg Br 1971;53:429-39.

14. Fowles JV, Kassab MT. Observations concerning radial neck fractures in children. J Pediatr Orthop 1986;6:51-7.

15. Kaufman B, Rinott MG, Tanzman M. Closed reduction of fractures of the proximal radius in children. J Bone Joint Surg Br 1989;71:66-7.

16. Monson R, Black B, Reed M. A new closed reduction technique for the treatment of radial neck fractures in children. J Pediatr Orthop 2009;29:243-7.

17. Neher CG, Torch MA. New reduction technique for severely displaced pediatric radial neck fractures. J Pediatr Orthop 2003;23:626-8.

18. Schmittenbecher PP, Haevernick B, Herold A, Knorr P, Schmid E. Treatment decision, method of osteosynthesis, and outcome in radial neck fractures in children: a multicenter study. J Pediatr Orthop 2005;25:45-50.

19. Tan BH, Mahadev A. Radial neck fractures in children. J Orthop Surg (Hong Kong) 2011;19:209-12.

20. Steele JA, Graham HK. Angulated radial neck fractures in children: a prospective study of percutaneous reduction. J Bone Joint Surg Br 1992;74:760-4.

21. Evans MC, Graham HK. Radial neck fractures in children: a management algorithm. J Pediatr Orthop B 1999;8:93-9.

22. Bernstein SM, McKeever P, Bernstein L. Percutaneous reduction of displaced radial neck fractures in children. J Pediatr Orthop $1993 ; 13: 85-8$

23. Valone LC, Waites C, Tartarilla AB, et al. Functional elbow range of motion in children and adolescents. J Pediatr Orthop 2020; 40:304-9. 